\title{
Symmetry of standing waves generated by a point defect in epitaxial graphene
}

\author{
L. Simon ${ }^{1}$, C. Bena ${ }^{2}$, F. $\operatorname{Vonau}^{1}$, D. Aubel ${ }^{1}$, H. Nasrallah ${ }^{1}$, M. Habar ${ }^{1}$, J. C. Perruchetti ${ }^{1}$ \\ ${ }^{1}$ Laboratoire de Physique et de Spectroscopie Electronique \\ CNRS-UMR7014, 4, rue des Frères Lumière 68093 Mulhouse-France \\ 2 Institut de Physique Théorique, CEA/Saclay, CNRS-URA 2306 \\ Orme des Merisiers, F-91191 Gif-sur-Yvette, France
}

(Dated: October 30, 2018)

\begin{abstract}
Using scanning tunneling microscopy (STM) and Fourier Transform STM (FT-STM), we have studied a point defect in an epitaxial graphene sample grown on silicon carbide substrate. This analysis allows us to extract the quasiparticle energy dispersion, and to give a first experimental proof of the validity of Fermi liquid theory in graphene for a wide range of energies from -800 $m e V$ to $+800 \mathrm{meV}$. We also find evidence of a strong threefold anisotropy in the standing waves generated by the defect. We discuss possible relations between this anisotropy, the chirality of the electrons, and the asymmetry between graphene's two sublattices. All experimental measurements are compared and related to theoretical T-matrix calculations.
\end{abstract}

PACS numbers: 68.65.-k, 81.16.Fg, 81.07.-b, 81.16.Rf, 82.30.RS, 82.65.+r

Graphene is a two-dimensional zero-gap semiconductor that shows many fascinating physical properties, in particular the quasiparticles near the Dirac point have a massless spectrum and linear dispersion. These relativistic particles have a velocity of about one percent of the speed of light, and are able to propagate ballistically over mesoscale distances [1]. The two inequivalent $\mathrm{A}$ and $\mathrm{B}$ sublattices of the $2 \mathrm{D}$ honeycomb carbon structure give rise to the fermionic pseudospin and chirality that lie at the origin of the forbidden backscattering in the presence of extended impurities [2], and of the anomalous Friedel oscillations in the vicinity of localized impurities [3]. The possibility to make few-layer graphene samples by the annealing of a silicon carbide substrate has been previously demonstrated in Refs. [4, 5, 6]. The Dirac fermion behavior has been evidenced in epitaxial graphene by angleresolved photoemission spectroscopy (ARPES) [7, 8].

Here we analyze the Fourier transform of STM conductance images in bilayer epitaxial graphene. It has been shown that such a study should allow one to extract the quasiparticle dispersion. This was first observed in superconductors [9], then by some of the authors in a semi-metallic material 10, 11], and more recently in bilayer graphene [12]; the latter experiment was however limited to a small energy window $( \pm 100 \mathrm{meV})$. Various other STM observations of the LDOS distortions in the presence of impurities in few-layer graphene exist in the literature (see for example [13, 14, 15, 16], for hydrogeninduced defects on graphite surfaces, and [12, 17] for several types of defects in graphene bilayers).

In this Letter we present an in-depth theoretical (single impurity T-matrix) and experimental (STM and FTSTM) study of the local density of states (LDOS) in the

\footnotetext{
${ }^{*}$ corresponding author

Email address: L.Simon@uha.fr
}

vicinity of an impurity. We have looked for point defects and we have focused on a single isolated impurity that strongly affects the LDOS of the epitaxial graphene layer; the distortion induced by this defect turns out to have a threefold symmetry. Using FT-STM we test the robustness of the quasiparticle dispersion, and the range of validity of the Fermi liquid theory in bilayer (or multilayer) graphene for a large energy window $(-800$ to +800 $m e V)$. Our experiment was performed with a LT-STM from Omicron at $77 \mathrm{~K}$ in a base pressure of $10^{-11}$ mbar. The samples were prepared in UHV by the annealing of n-doped $\mathrm{SiC}(0001)$ at $1000 \mathrm{~K}$ starting from a $3 \times 3$ reconstruction [5].

Figure 11A shows a topographic image of a large graphene terrace taken at $-17 \mathrm{meV}$ (probing full states). This layer shows an intriguing "star-like" defect with an apparent six-fold $(C 6 v)$ symmetry. This atomic defect is accompanied by a strong distortion of the graphene lattice. The center appears black, which is a dramatic change from the case of the unperturbed lattice. As schematized in figure $1 \mathrm{C}$, a detailed analysis of the realspace image, shows that the point defect is directly located over or under a lattice site. The 2D Fourier transform of the topographic image is shown in $1 \mathrm{E}$. Six highintensity regions corresponding to the periodicity of the hexagonal lattice appear at the centers $\Gamma$ of the six Brillouin zones (BZ) neighboring the first BZ. Six highintensity features (open circles) with an anisotropic intensity are also observed around the corners of the first BZ. They can be attributed to the scattering of quasiparticles between isocontours surrounding the $\mathrm{K}$ points (the corners of the BZ) as schematized in figure 1D. This type of scattering is called inter-nodal or inter-valley scattering. The six high-intensity regions at the corners of the first BZ correspond to scattering processes that link two consecutive isocontours. Similar features have also been observed in [12, 14, 17, 18].

Using FFT filtering [19] we have removed the lattice

Typeset by REVTEX 


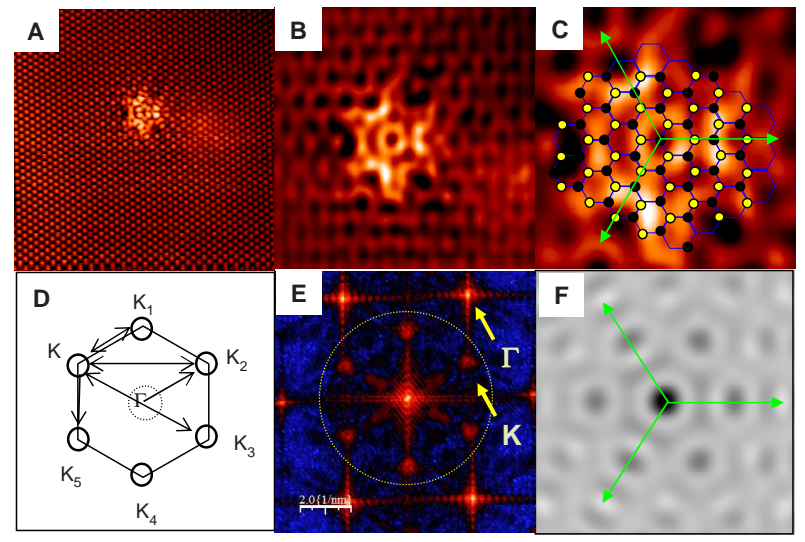

FIG. 1: A) STM topographic image $\left(10 \times 10 \mathrm{~nm}^{2},-17 \mathrm{meV}\right.$ $1 n A)$ showing an isolated defect. B) Zoom-in $\left(5 \times 5 \mathrm{~nm}^{2}\right)$ or the defect, subsequent to a FFT filtering removing the atomic resolution and all other features with wavevectors outside the yellow circle in E. C) $2.5 \times 2.5 \mathrm{~nm}^{2}$ zoom in on B with the schematic atomic lattice of graphene (A/black and B/yellow atoms) superimposed over the standing-wave pattern arounc the point defect. D) schematic representation of the 2D Brillouin zone and of the constant energy contours, indicating the possible scattering processes. E) The FFT power spectrum o: the $2 \mathrm{D}$ topographic image in A. F) The real-space LDOS in $\varepsilon$ bilayer graphene calculated for a point defect using a singleimpurity T-matrix approximation at $300 \mathrm{meV}$ above the Dirac point.

periodicity vectors and all other features with wavevectors outside the yellow circle in Fig. 1 $\mathrm{E}$, thus taking into account only the intra-valley and the inter-consecutivevalley scattering processes ( $K \rightarrow K_{1}$ type) depicted in figure 1D. The resulting real-space image is shown in figure 1B. This operation strongly enhances the anisotropic intensity observed also on the bare topographic image. The LDOS near the defect shows a clear threefold $(C 3 v)$ symmetry. While, as depicted in Fig. 1C, close to the impurity we observe a fairly homogeneous standing-wave ring, with an almost perfect six-fold symmetry, farther away from the impurity, the intensity is clearly higher along three axes (drawn in green in Figs. $1 \mathrm{C}$ and $1 \mathrm{~F}$ ). direction.

Let us now analyze the possible origin of this threefold symmetry. If the defect is localized above an A atom (shown in black in Fig. 1C), the nearest neighbors are three B atoms ( $C 3 v$ symmetry). The second-nearest neighbors are six A atoms (C6v symmetry). Hence, the three-fold symmetry comes from the lattice environment, in particular from the scattering of electrons between the $\mathrm{A}$ and the B sublattices. This is related to the pseudospin and chirality of electrons in graphene and will be explained in more detail in what follows.

The theoretical LDOS modulation for a bilayer graphene in the presence of a single impurity is represented in Fig. 1F. This was obtained at an energy of

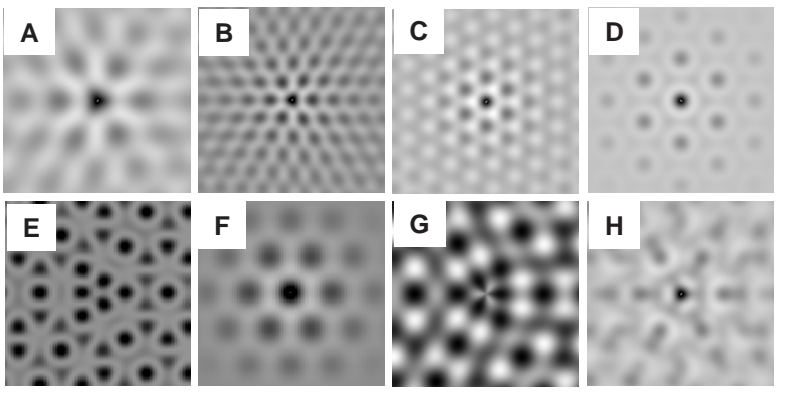

FIG. 2: Calculated real-space LDOS modulations in a monolayer graphene for a point defect placed on top of an A atom. Figs. 2 A, B, C depict the contributions of selected scattering processes to the LDOS $\left(K \rightarrow K_{1}, K \rightarrow K_{2}\right.$ and $K \rightarrow K_{3}$ respectively) as indicated in Fig. 1D; in $2 \mathrm{~F}$, and $2 \mathrm{G}$ we depict the separate contributions of the $\mathrm{A}$ and $\mathrm{B}$ sublattices to the $K \rightarrow K_{1}$ LDOS modulations in 2A. Figs. 2D and E depict the $\mathrm{A}$ and $\mathrm{B}$ sublattice contributions to the full LDOS, evaluated when all scattering processes are considered. In $\mathrm{H}$ we depict the sum of the $\mathrm{A}$ and $\mathrm{B}$ contributions to the full LDOS, when the weight of the $K \rightarrow K_{1} \mathrm{~B}$ component is increased three times compared to that of the $K \rightarrow K_{1}$ A component.

$300 \mathrm{meV}$ above the Fermi level, using the formalism presented in [20]. One can observe quite a few features similar to the ones depicted in Fig. 11C, including the existence of a three-fold symmetry. However the three-fold anisotropy is much less pronounced.

Figure2 2 displays the calculated real-space modulations in the LDOS close to the point defect, when only specific scattering processes have been considered. For simplicity this calculation has been performed for a monolayer graphene, as for bilayer graphene it is less clear how much the two bands and the two layers contribute to the measured LDOS. The intra-nodal scattering processes are responsible for radially-symmetric features with small $\vec{q}$ wavevectors and should therefore not be responsible for the presence of the three-fold symmetry. Hence we do not focus on these modulations here.

In Figs. 22A, 2B and 22 C, we plot the LDOS modulations due solely to scattering between nearest, second-nearest, and third-nearest neighboring nodes, generically denoted $K \rightarrow K_{1}, K \rightarrow K_{2}$ and $K \rightarrow K_{3}$ respectively, as schematized in Fig. 1D. First, it is clear that the most important effect of the impurity, which is responsible for the $R 3$ patterns observed experimentally, is the $K \rightarrow K_{1}$ scattering. Second, close to the impurity, the patterns are almost sixfold symmetric, with a slight three-fold anisotropy. Thus, none of these scattering events contributes significantly to the threefold symmetry. To investigate the source of the anisotropy, we first note that, in Ref. [3] it was shown that the $K \rightarrow K_{1}$ high-intensity regions appearing in the FT of the LDOS at the corners of the BZ are anisotropic, more pronouncedly for monolayer graphene than for bilayer graphene. This is due to the chirality of the quasi- 
particles in graphene. We believe that this asymmetry in the observed momentum space features, and thus the chirality of quasiparticles, is responsible for the small amount of $C 3 \mathrm{v}$ asymmetry observed in the theoretical real-space LDOS calculations. The second observation is that the anisotropy comes mainly from the B component of the LDOS, that describes the double virtual hopping of quasiparticles between the A and B sublattices[21]. This is detailed in Figs. $2 \mathrm{~F}$ and $2 \mathrm{G}$, where we plot separately the $\mathrm{A}$ and the $\mathrm{B}$ sublattice contributions to the LDOS modulations generated by $K \rightarrow K_{1}$ scattering. We can see that the contribution from the A atoms is fully sixfold symmetric, while the contribution of the B atoms is three-fold symmetric. This is also true for the A and $\mathrm{B}$ components (depicted in $2 \mathrm{D}$ and $2 \mathrm{E}$ respectively) of the full LDOS obtained from summing the contributions of all scattering processes. Fig. $2 \mathrm{H}$ depicts the sum of the $\mathrm{A}$ and $\mathrm{B}$ contributions to the full LDOS, where in order to increase the anisotropy, we have multiplied the $K \rightarrow K_{1} \mathrm{~B}$ contribution by three. This may mimic bilayer graphene where, due to the coupling between the two layers, the sublattices A and B may not contribute equally to the observed LDOS. A very strong threefold symmetry is observed in this weighted superposition, and the resulting image corresponds more closely to the pattern observed experimentally in figure $1 \mathrm{~B}$ ). This is consistent with having a defect at an A site whose dominant effect is the scattering of sublattice $B$ electrons between two consecutive valleys $\left(K \rightarrow K_{1}\right)$.

We are also interested in how such a defect affects the relativistic character of the quasiparticles. To find this, we measured the quasiparticle energy dispersion using the Fourier transform of conductance images at different energies. Every image was acquired using a lock-in amplifier and a modulation voltage of $\pm 20 \mathrm{meV}$ which gave the energy uncertainty. We have performed a 2D FFT of these images, and in Figs. $3 \mathrm{~A}$ and $3 \mathrm{~B}$ we show the resulting intensity profiles along the direction $K-\Gamma-K$. For a wide range of energies, between energies well below the Fermi level $(-950 \mathrm{meV})$ to about $-150 \mathrm{meV}$, the width of the peak at the center of the Brillouin zone (measured at half maximum) shows a clear dispersion with the bias voltage (it decreases when the energy moves towards the Fermi level). At energies closer to the Fermi level the profile becomes more complex and displays a dip in the intensity profile close to the $\Gamma$ point. The central peak also shows two lateral structures (shoulders). The width of the central feature does not appear to disperse with energy close to the Dirac point, but it starts dispersing again for higher positive energies. In Fig. $3 \mathrm{C}$ we show the STS measurement of the LDOS $(d I / d V$ as a function of bias voltage) taken close to the impurity. We can see a $150 \mathrm{meV}$ gap-like feature centered around the Fermi level at $0 \mathrm{meV}$. Fig. 4 shows a linear dependence of the width of the central feature with energy, except in an energy range from $-200 \mathrm{meV}$ to $100 \mathrm{meV}$. For

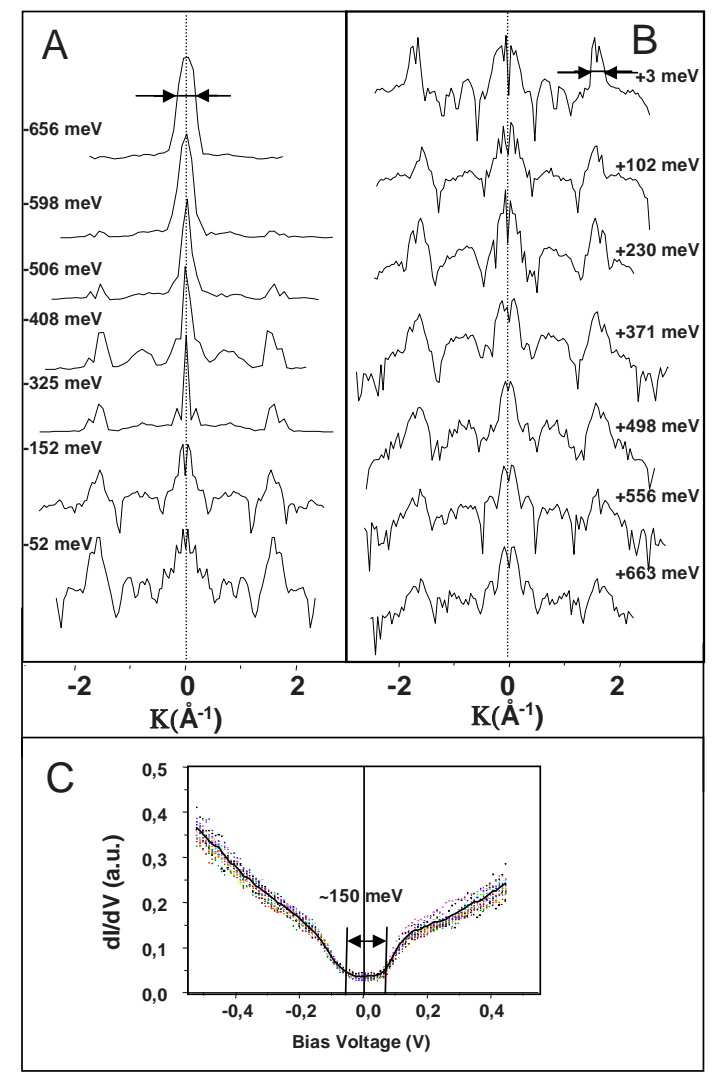

FIG. 3: A) and B) Intensity profiles along the $K-\Gamma-K$ direction of the 2D FT of the LDOS taken for different bias voltages. C) STS measurement taken near the impurity showing a gap centered around the Fermi level.

these energies the shape of the central peak is more complex. The width of the features at the $\mathrm{K}$ points follows a similar dispersion. Beyond $+500 \mathrm{meV}$, all the features seem to follow a different dispersion branch, as indicated in Fig. 4. We estimate the Dirac point at $-100 \mathrm{meV}$ below the Fermi level. The spreading in the dispersion of the points close to the Fermi level could be attributed to the presence of the gap. We compare the experimental results with similar theoretical profiles obtained using a T-matrix approximation for a single localized impurity in bilayer graphene [3]. No gap at the Fermi level was included in the theoretical calculation, while a small gap of $\approx 100 \mathrm{meV}$ was assumed near the Dirac point, which was taken to be close to $-250 \mathrm{meV}$. In Fig. $4 \mathrm{~B}$ we plot the dispersion of the central and K-points features, and of the shoulder, obtained from the theoretical curves. We also observe the presence of two different dispersion branches. The second dispersion branch and the shoulder arise because of the bilayer graphene upper band which opens at energies higher than the inter-layer coupling. For monolayer graphene these extra features should not appear.

In conclusion, our measurements show that the quasiparticle approximation and the Fermi liquid theory are 


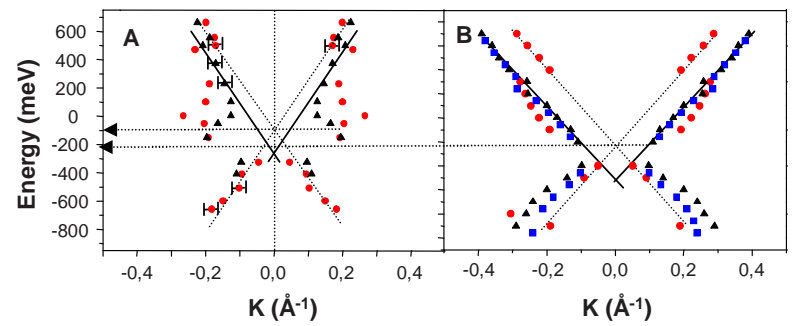

FIG. 4: The dispersion for the width of the central-ring feature (red circles) and of the K-points feature (black triangles) obtained from the experimental data (A), and from the theoretical $K-\Gamma-K$ cuts (B). The dispersion of the centralfeature shoulder on the theoretical curves is indicated by blue squares.

robust over a large range of energies. The complex structure of the central feature (the existence of the shoulder), as well as the presence of two dispersion branches are consistent with the bilayer (or multilayer) character of the graphene sample. While the point defect modifies the electronic wave-function in its vicinity, a clear linear dispersion is still observed, and the relativistic character of the quasiparticle is preserved. Also, the STS measurements in Fig. $3 \mathrm{C}$ indicate the presence of a gap centered at the Fermi point, and not at the Dirac point inferred from our FT-STM measurements. This gap is thus different from the Dirac-point gap observed by ARPES [7, 8] which was attributed to the different doping levels of the epitaxial graphene layers. It is however consistent with previous STM measurements performed on epitaxial graphene [22], and more recently on exfoliated graphene [23], where a gap observed at the Fermi level was attributed to a pinning of the tunneling spectrum due to the coupling with phonons.

A marked three-fold symmetry in the vicinity of the point defect was also observed. Detailed theoretical studies of the different scattering events underline the importance of the scattering between consecutive nodal points $\left(K \rightarrow K_{1}\right)$. Our analysis also suggests that for bilayer graphene, the two sublattices do not contribute equally to the measured LDOS. Thus, the amount of asymmetry observed in the LDOS near the impurity is consistent with the fact that the scattering of the electrons between the sites of the sublattice on which the impurity is located (A) and the electrons on the B sublattice dominate over the scattering of the electrons between sites of the same (A) sublattice.

We would like to thank P. Y. Clement for useful discussions. This work is supported by the Région Alsace and the CNRS. C. Bena acknowledges the support of a Marie Curie Action under FP6.
[1] K. S. Novoselov, A. K. Geim, S. V. Morozov, D. Jiang, M. I. Katsnelson, I. V. Grigorieva, S. V. Dubonos, and A. A. Firsov, Nature 438197 (2005).

[2] V. V. Cheianov and V. I. Fal'ko, Phys. Rev. Lett. 97, 226801 (2006); E. Mariani et. al., cond-mat/0702019; T. Ando, J. Phys. Soc. Japan 75074716 (2006); M. I. Katsnelson, K. S. Novoselov and A. K. Geim, Naturephysics 2620 (2006).

[3] C. Bena, Phys. Rev. Lett. 100076601 (2008).

[4] I. Forbeaux, J.-M. Themlin and J.-M. Debever, Phys. Rev. B 5816396 (1998).

[5] L. Simon, J. L. Bischoff and L. Kubler, Phys. Rev. B 60 11653 (1999).

[6] C. Berger, Z. Song, T. Li, X. Li, A. Y. Ogbazghi, R. Feng, Z. Dai, A. N. Marchenkov, E. H. Conrad, P. N. First, W. A. de Heer, J. Phys. Chem., 108, 19912 (2004).

[7] A. Bostwick, T. Ohta, T. Seyller, K. Horn and E. Rotenberg, Naturephysics, 336 (2007); A. Bostwick et.al., arXiv:0705.3705

[8] S.Y. Zhou, G.-H. Gweon, A. V. Fedorov, P. N. First, W. A. de Heer, D.-H. Lee, F. Guinea, A. H. Castro Neto and A. Lanzara, Nature Mat. 6770 (2007).

[9] K. McElroy, R. W. Simmonds, J. E. Hoffman, D.-H. Lee, J. Orenstein, H. Elsaki, S. Uchida and J. C. Davis, Nature, 422592 (2003).

[10] F. Vonau, D. Aubel, G. Gewinner, S. Zabrocki, J. C. Peruchetti, D. Bolmont and L. Simon, Phys. Rev. Lett. 95176803 (2005).

[11] L. Simon, F. Vonau and D. Aubel, J. of Phys. Cond. Mat.,19 355009 (2007).

[12] G. M. Rutter, J. N. Crain, N. P. Guisinger, T. Li., P. N. First and J. A. Stroscio, Science 317219 (2007).

[13] P. Ruffieux, M. Melle-Franco, O. Gröning, M. Bielmann, F. Zerbetto, and P. Gröning, Phys. Rev. B 71153403 (2005).

[14] P. Ruffieux, O. Gröning, P. Schwaller, L. Schlapbach and P. Gröning, Phys. Rev. Lett. 844910 (2000).

[15] K. F. Kelly and N.J. Halas, Surf. Science 416 L108 (1998).

[16] Y. Niimi, H. Kambara, T. Matsui, D. Yoshioka and H. Fukuyama Phys. Rev. Lett. 97236804 (2006).

[17] P. Mallet, F. Varchon, C. Naud, L. Magaud, C. Berger and J. Y. Veuillen, Phys. Rev. B 76 041403(R) (2007).

[18] I. Brihuega, P. Mallet, C. Bena, S. Bose, C. Michaelis, L.Vitali, F. Varchon, L. Magaud, K. Kern, J. Y. Veuillen, arXiv:0806.2616

[19] We use the WsxM program, I. Horcas et al, Rev. Sci. Instrum. 78013705 (2007), applying a 2D FFT, and displaying a logarithmic scale power spectrum.

[20] C. Bena, arXiv:0807.1981,

[21] In contrast, the A component of the LDOS describes the hopping of quasiparticles between atoms on the A sublattice. This give rise to symmetric circles close to the corners of the BZ in the FT of the LDOS and to completely symmetric $C 6 v$ features in the real space.

[22] V. W. Brar, Y. Zhang, Y. Yayon and T. Ohta Appl. Phys. Lett. 91122102 (2007).

[23] Y. Zhang, V. W. Brar, F. Wang, C. Girit, Y. Yayon, M. Panlasigui, A. Zettl, M. F. Crommie http://arxiv.org/0802.4315 